Article

\title{
Improvement of Nanostructured Polythiophene Film Uniformity Using a Cruciform Electrode and Substrate Rotation in Atmospheric Pressure Plasma Polymerization
}

\author{
Jae Young Kim ${ }^{1,+}+\mathbb{D}$, Hyo Jun Jang ${ }^{1,+}$, Gyu Tae Bae ${ }^{1}$, Choon-Sang Park ${ }^{2}$, Eun Young Jung ${ }^{1, *}$ \\ and Heung-Sik Tae ${ }^{1,3, *}$
}

1 School of Electronic and Electrical Engineering, College of IT Engineering, Kyungpook National University, Daegu 41566, Korea; jyk@knu.ac.kr (J.Y.K.); bs00201@knu.ac.kr (H.J.J.); doctor047@knu.ac.kr (G.T.B.)

2 Department of Electrical and Computer Engineering, College of Engineering, Kansas State University, Manhattan, KS 66506, USA; purplepcs@ksu.edu

3 School of Electronics Engineering, College of IT Engineering, Kyungpook National University, Daegu 41566, Korea

* $\quad$ Correspondence: eyjung@knu.ac.kr (E.Y.J.); hstae@ee.knu.ac.kr (H.-S.T.); Tel.: +82-53-950-6563 (H.-S.T.)

+ These authors contributed equally to this work.

\section{check for} updates

Citation: Kim, J.Y.; Jang, H.J.; Bae, G.T.; Park, C.-S.; Jung, E.Y.; Tae, H.-S. Improvement of Nanostructured Polythiophene Film Uniformity Using a Cruciform Electrode and Substrate Rotation in Atmospheric Pressure Plasma Polymerization. Nanomaterials 2022, 12, 32. https:// doi.org/10.3390/nano12010032

Academic Editors: Carles Corbella and Sabine Portal

Received: 1 November 2021

Accepted: 21 December 2021

Published: 23 December 2021

Publisher's Note: MDPI stays neutral with regard to jurisdictional claims in published maps and institutional affiliations.

Copyright: (c) 2021 by the authors. Licensee MDPI, Basel, Switzerland. This article is an open access article distributed under the terms and conditions of the Creative Commons Attribution (CC BY) license (https:/ / creativecommons.org/licenses/by/ $4.0 /)$.

\begin{abstract}
In atmospheric pressure (AP) plasma polymerization, increasing the effective volume of the plasma medium by expanding the plasma-generating region within the plasma reactor is considered a simple method to create regular and uniform polymer films. Here, we propose a newly designed AP plasma reactor with a cruciform wire electrode that can expand the discharge volume. Based on the plasma vessel configuration, which consists of a wide tube and a substrate stand, two tungsten wires crossed at 90 degrees are used as a common powered electrode in consideration of two-dimensional spatial expansion. In the wire electrode, which is partially covered by a glass capillary, discharge occurs at the boundary where the capillary terminates, so that the discharge region is divided into fourths along the cruciform electrode and the discharge volume can successfully expand. It is confirmed that although a discharge imbalance in the four regions of the AP plasma reactor can adversely affect the uniformity of the polymerized, nanostructured polymer film, rotating the substrate using a turntable can significantly improve the film uniformity. With this AP plasma reactor, nanostructured polythiophene (PTh) films are synthesized and the morphology and chemical properties of the PTh nanostructure, as well as the PTh-film uniformity and electrical properties, are investigated in detail.
\end{abstract}

Keywords: atmospheric pressure plasma polymerization; conjugated polymer film; plasma polymerization; plasma synthesis; polythiophene nanostructure

\section{Introduction}

Atmospheric pressure (AP) plasma polymerization is a synthesis method in which vaporized monomer molecules successively interact with charged particles and excited species in nonthermal plasma at AP to generate various radicals and reactive species, react with each other, and form various polymers [1-7]. In nonthermal AP plasma, which is a partially ionized gas containing a large number of reactive, neutral, and charged particles, the plasma energy is mainly directed toward electrons with high mobility, and the ions and neutral species in the plasma medium remain at room temperature [8-14]. Thus, in nonthermal AP plasma, diverse reactive species and high-energy electrons exist at low gaseous temperatures, and this nonthermal behavior has great potential for applications in synthesizing thermally sensitive materials, including polymers. AP plasma polymerization is a convenient and effective approach for depositing conjugated nanostructured polymer films, because this process has the great advantage of not requiring a vacuum chamber and the relevant vacuum equipment for plasma polymerization [15-18]. 
In our previous reports, the device configuration of a guided wide tube and a substrate stand have been proposed to effectively block ambient air during AP plasma polymerization and to make the reactive monomer species stay longer inside the plasma reactor [19-22]. By confining the plasma medium using the guided tube and the substrate stand, the spatial uniformity of the discharge gas and the monomer vapor could be ensured for a longer period of time, enabling the intensive deposition of nanostructured polymeric films onto the substrate. Our research group recently reported on low-voltage-driven AP plasma polymerization using a bare electrode, in which the electrode was exposed to the plasma medium $[23,24]$. The AP plasma reactor with a pin-type bare electrode did not require charging and discharging of the capacitive dielectric barrier and, thus, the AP plasma could be generated at a lower voltage. This advantage also made it possible to use higher amounts of monomers in the polymerization process even during low-power plasma operation. However, the drawback of this AP plasma reactor is that it has a structural limitation in securing a uniform and wide polymerization processing area in spite of structural optimization efforts, because it uses a single pin-shape electrode. In order to apply the polymer film deposited by the AP plasma polymerization method to various research and industrial fields, it is important to ensure the film uniformity of the synthesized polymer films. Therefore, it is necessary to develop a new AP plasma reactor that can more easily expand the processing area in two dimensions.

In this study, we propose a new AP plasma reactor with a cruciform (cross-shaped)bare-electrode structure in which two bare wires are crossed laterally in two dimensions, and optimize the experimental conditions to obtain uniform and wide nanostructured polythiophene (PTh) films in the range of several square centimeters. In particular, AP plasma reactors are fabricated with different lengths of tungsten wire exposed to the plasma space, and the discharge characteristics and PTh-film uniformity are examined with respect to the bare length of the electrode. In addition, AP plasma polymerization is performed while rotating the substrate stand using a turntable in order to obtain a uniformly nanostructured PTh film.

\section{Materials and Methods}

\subsection{Experimental Setup for AP Plasma Polymerzation}

A schematic diagram of the AP plasma polymerization system employed in this study is presented in Figure 1. The gas feedline was divided into two units for independent control of the gas flow rates for AP plasma generation and vaporized monomer injection. Argon (Ar) gas (HP grade with a purity of 99.999\%) was used as the discharge gas at a flow rate of 1000 standard cubic centimeters per minute $(\mathrm{sccm})$. Liquid thiophene monomer with a molecular weight of $84.14 \mathrm{~g} \cdot \mathrm{mol}^{-1}$ (Sigma-Aldrich Co., St. Louis, MO, USA) was vaporized using a glass bubbler supplying an Ar flow rate of $100 \mathrm{sccm}$. Using an invertertype driving circuit, a sinusoidal high voltage with a peak value of $10 \mathrm{kV}$ and a frequency of $28 \mathrm{kHz}$ was applied to the powered electrode of the AP plasma reactor. A turntable (T10, Excam, Busan, Korea) was used to rotate the substrate stand of the AP plasma reactor.

To monitor the electrical characteristics of the generated plasma, the voltage and current waveforms from the powered electrode were displayed on a digital oscilloscope (TDS 3014B, Tektronix Inc., Beaverton, OR, USA) using a high-voltage probe (P6015A, Tektronix Inc., Beaverton, OR, USA) and a current transformer (4100, Pearson Electronics Inc., Palo Alto, CA, USA).

Photographs of the AP plasma reactor and the glass disk substrates were obtained using a digital single-lens reflex camera (D5300, Nikon Corp., Tokyo, Japan) equipped with a Macro 1:1 lens (Tamron SP AF 90 mm F2.8 Di, Tamron Co., Ltd., Saitama, Japan), and an intensified charge-coupled device (ICCD) camera (PI-MAX II, Princeton Instruments Inc., Trenton, NJ, USA) was used in shutter mode to identify the spatial distribution of the generated glow plasma.

For iodine $\left(\mathrm{I}_{2}\right)$ doping, nanostructured PTh films deposited onto gold interdigitated electrode (IDE)-patterned silicon (Si) substrates were placed in a container with $2 \mathrm{~g}$ of 
solid $\mathrm{I}_{2}$ pellets (Sigma-Aldrich Co., St. Louis, MO, USA, 99.99\%) and vacuum sealed for 30 min using a vacuum sealer packaging machine (VP-5700, Intropack, Pyeongtaek, Korea). The IDE had an interdigitated comb-shaped two-electrode structure with 20 pairs of microelectrodes. The width of the IDE was $10.8 \mu \mathrm{m}$, and the spacing between the adjacent IDEs was $2.54 \mu \mathrm{m}$.

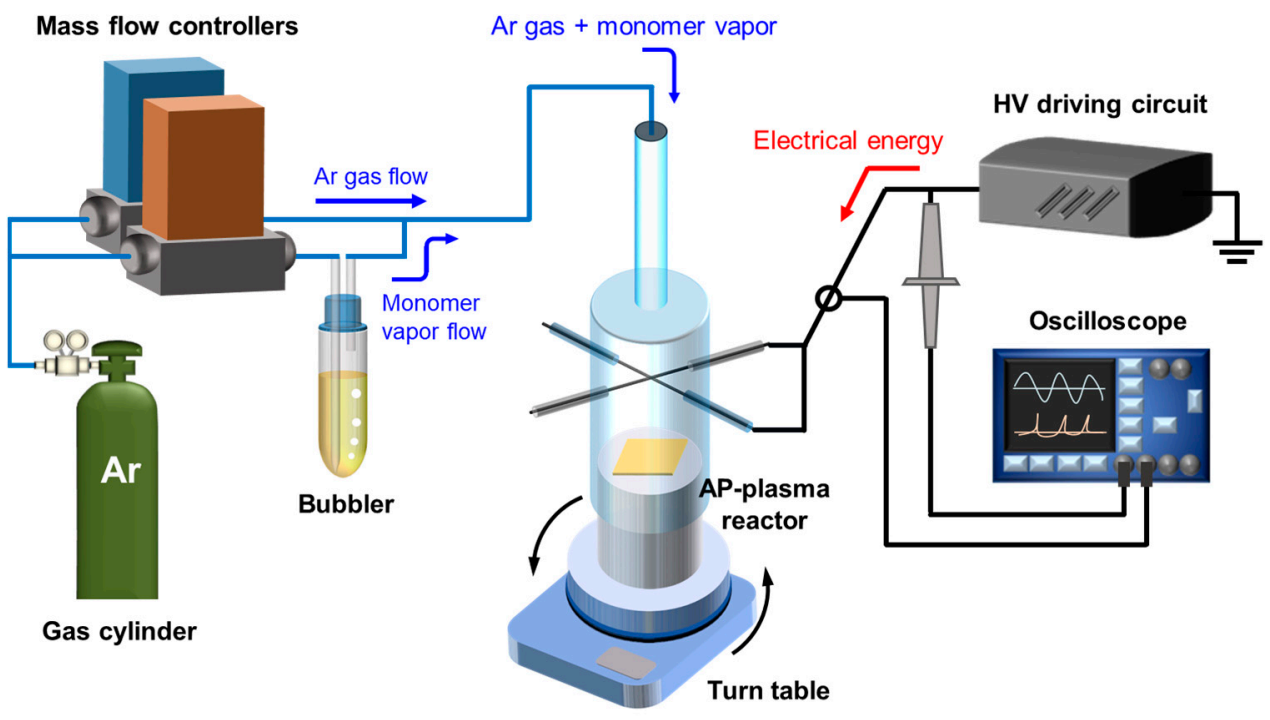

Figure 1. Schematic diagram of the AP plasma polymerization system combined with gas supply, AP plasma reactor, and high-voltage power supply.

\subsection{Analysis and Characterization of the Nanostructured Polythiophene Film}

The surface and cross-sectional morphology, thickness, and vertical orientation of the nanostructured PTh films on Si substrates were observed using field emission scanning electron microscopy (FE-SEM; SU8220, Hitachi High-Technologies, Tokyo, Japan) with accelerated electrons at a voltage of $3 \mathrm{kV}$ and a current of $10 \mathrm{~mA}$. The samples were coated with platinum to avoid surface-charging problems during analysis.

The thickness profile of the nanostructured PTh film was measured at 15 positions with an interval of $2 \mathrm{~mm}$ using a stylus profiler (P-7, KLA Tencor Corp., Milpitas, CA, USA) at the Korea Basic Science Institute (KBSI, Busan, Korea). Measurements were performed while moving the stylus in contact with the PTh-film surface at a scan speed of $200 \mu \mathrm{m} \cdot \mathrm{s}^{-1}$.

The chemical composition of the PTh films synthesized under different conditions were compared via Fourier-transform infrared spectroscopy (FTIR; Vertex 70, Bruker, Ettlingen, Germany) at KBSI (Daegu, Korea). The attenuated total reflection (ATR) FTIR spectra were measured by averaging 128 scans in the range of 650 to $4000 \mathrm{~cm}^{-1}$ at a wavenumber resolution of $0.6 \mathrm{~cm}^{-1}$.

High-resolution transmission electron microscopy images were taken with a Titan G2 ChemiSTEM Cs Probe (FEI Company, Hillsboro, OR, USA) transmission electron microscope operating at $200 \mathrm{kV}$. A transmission electron microscopy (TEM) sample of PTh nanoparticles was prepared by depositing a $10-\mu \mathrm{L}$ solution (ultrasonically dispersed in DI water) onto carbon-coated copper grids and drying in air.

The X-ray photoelectron spectroscopy (XPS; ESCALAB 250XI, Thermo Fisher Scientific, Waltham, MA, USA) was carried out in order to investigate the surface chemical compositions, using monochromatic Al $\mathrm{K} \alpha$ X-ray source $(\mathrm{h} v=1486.71 \mathrm{eV})$ operated at $15 \mathrm{kV}$ and $20 \mathrm{~mA}$. The pressure in the analyzing chamber was maintained at $10^{-8} \mathrm{~Pa}$ or lower during analysis and the size of the analyzed area was $500 \mu \mathrm{m} \times 500 \mu \mathrm{m}$. Spectra were acquired with the angle between the direction of the emitted photoelectrons and the surface equal to $60^{\circ}$. The estimated analyzing depth of the used XPS setup was 8 to $10 \mathrm{~nm}$. The high-resolution spectra were taken in the constant-analyzer-energy mode with $200 \mathrm{eV}$ for the survey scan and a $50 \mathrm{eV}$ pass energy for the element scan. The value of $285.5 \mathrm{eV}$ 
of the $C$ 1s core level was used for the calibration of the energy scale. To curve fit the high-resolution $C 1 s, S 2 p$, and $O 1$ s peaks, the deconvolution of the $C 1 s, S 2 p$, and $O$ 1s peaks was analyzed by the Thermo Advantage software. The peaks were deconvoluted using Gaussian-Lorentzian peak shapes (constrained between $80 \%$ and $100 \%$ Gaussian) and the full-width at half maximum (FWHM) of each line shape was constrained between 2.0 and $3.0 \mathrm{eV}$.

\section{Results and Discussion}

\subsection{Newly Designed AP Plasma Reactor with Cruciform Bare Electrode}

Unlike a pin-type bare electrode structure in which the electric field is locally concentrated at the end of the pin-type electrode due to its protruding shape [23-25], it is necessary to first check where the discharge occurs in a plasma reactor with a powered electrode in which the wire is positioned parallel to the substrate. The discharge behaviors were tested by placing a bare wire in the center of the plasma reactor and varying the bending angle of the wire protruding toward the substrate as depicted in Figure 2. A 0.5 mm-diameter tungsten wire was used as the power electrode and covered with a glass capillary, and $20 \mathrm{~mm}$ was left exposed in the center of the wire for plasma generation.

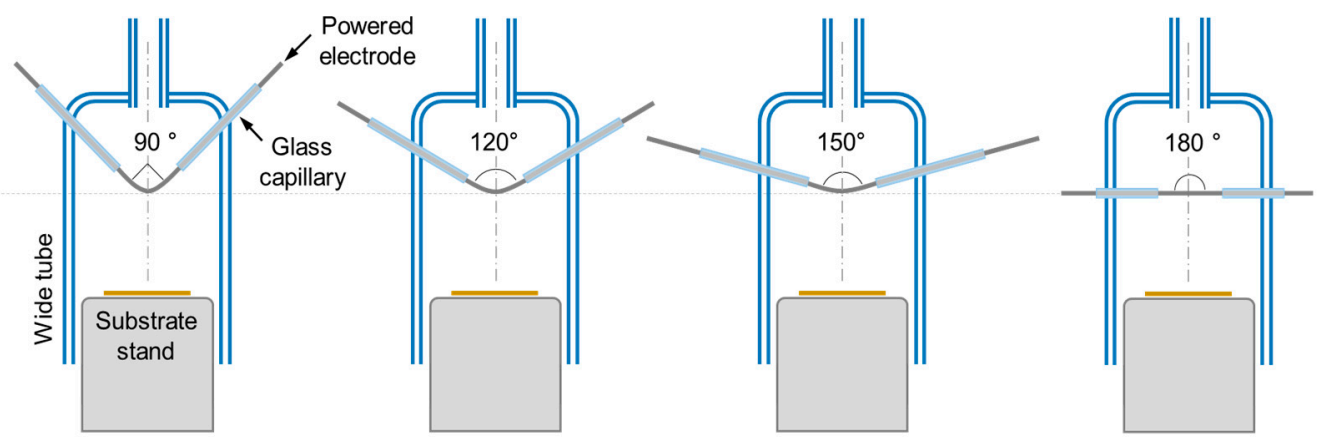

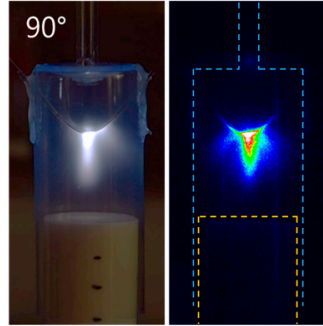

(a)

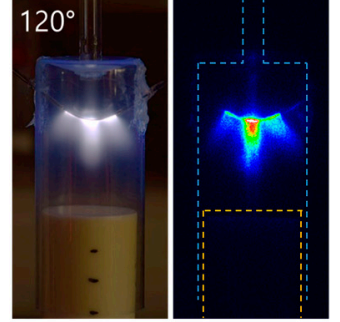

(b)

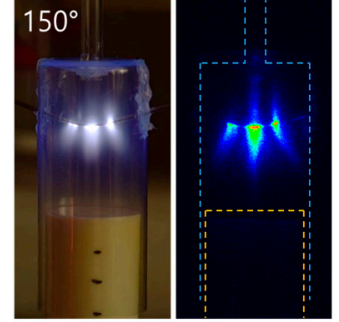

(c)

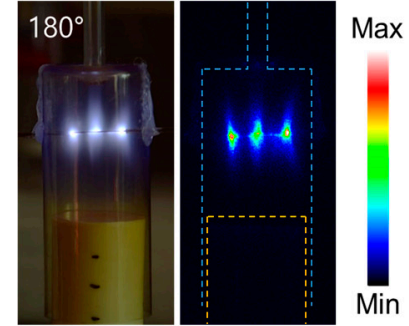

(d)

Figure 2. Optical observation (digital photographs and ICCD images) of glow plasma according to bending angle of tungsten wire electrode: (a) 90 degrees, (b) 120 degrees, (c) 150 degrees, and (d) 180 degrees.

As shown in Figure 2a, when the bare part of the wire was bent at 90 degrees, the discharge occurred only around the bending area similar to the discharge of a pin-type electrode. When the bare part of the electric wire was bent at 120 degrees, the discharge in the bending part at the center was weakened and the discharge spread to the entire bare electrode (Figure 2b). As the bending angle of the wire further increased to 150 and 180 degrees, the discharge at the central bending area gradually weakened, and two additional discharge regions were created at the boundaries of the bare wire and the glass capillary (Figure 2c,d). Therefore, a total of three discharge regions were formed inside the AP plasma reactor. As a result of this test, it was observed that the bending angle of the wire was very critical in determining the location and behavior of the discharge when the gas flow and operating voltage conditions were kept the same. In particular, when the bare part of the electric wire was not bent at all and, thus, there was no protruding part 
(Figure 2d), the discharge was strongly generated by localized electric-field enhancement at the two boundary regions between the bare wire and the glass capillary [26-28]. On the other hand, since the applied voltage did not change, the central discharge became weaker as the discharge intensity at the boundary increased.

To extend the experiments in Figure 2 to two dimensions, our research group developed an AP plasma reactor using two intersecting tungsten wires as a common power electrode. To designate the area where the discharge occurred in the AP plasma reactor, the intersection of the two wires was left as bare wire, and the rest of the electrode was covered with glass capillary tubes. The part where the two tungsten wires cross at 90 degrees was placed in the center of the wide tube and vertically aligned with the gas-emitting region. The detailed experimental conditions of this study are listed in Table 1. In addition, in this study, several parametric studies were performed in order to identify the optimal experimental conditions for depositing PTh films, which are summarized in Table 2.

Table 1. Summary of experimental conditions for AP plasma polymerization.

\begin{tabular}{llc}
\hline & Experimental Conditions & AP Plasma Reactor \\
\hline \multirow{2}{*}{ Device } & Powered electrode shape & Cruciform \\
Configuration & Electrode material & Tungsten wire \\
& Inner diameter of wide tube & $34 \mathrm{~mm}$ \\
& Diameter of substrate stand & $30 \mathrm{~mm}$ \\
& Distance between electrode and substrate & $30 \mathrm{~mm}$ \\
\hline \multirow{2}{*}{ Driving } & Voltage waveform & Sinusoidal \\
Conditions & Plasma initiation voltage $\left(\mathrm{V}_{\mathrm{p}}\right)$ & $4 \mathrm{kV}$ \\
& Plasma driving voltage $\left(\mathrm{V}_{\mathrm{p}}\right)$ & $10 \mathrm{kV}$ \\
& Driving frequency & $28 \mathrm{kHz}$ \\
& Averaged power 1 & $4.40 \mathrm{~W}$ \\
\hline & Discharge and monomer carrier gas & Ar $(\mathrm{HP}$ grade: $99.999 \%)$ \\
& Gas flow rate for AP plasma discharge & $1000 \mathrm{sccm}$ \\
& Gas flow rate for thiophene monomer vapor & $100 \mathrm{sccm}$ \\
& Polymerization process time & $10 \mathrm{~min}$
\end{tabular}

${ }^{1}$ Figure S1 provides waveforms of applied voltage, total current, power and discharge current.

Table 2. Summary of experimental conditions for parametric studies.

\begin{tabular}{|c|c|c|c|c|c|}
\hline \multicolumn{6}{|c|}{ Parametric Studies } \\
\hline Conditions & & & Variables & & \\
\hline Length of the bare-wire part of the electrode & $2,15,30 \mathrm{~mm}$ & $15 \mathrm{~mm}$ & $15 \mathrm{~mm}$ & $15 \mathrm{~mm}$ & $15 \mathrm{~mm}$ \\
\hline Distance between electrode and substrate & $30 \mathrm{~mm}$ & $\underline{30,40,50 \mathrm{~mm}}$ & $30 \mathrm{~mm}$ & $30 \mathrm{~mm}$ & $\underline{20 \mathrm{~mm}}$ \\
\hline Ar flow rate for AP plasma discharge & $1000 \mathrm{sccm}$ & $1000 \mathrm{sccm}$ & $1000 \mathrm{sccm}$ & $1000 \mathrm{sccm}$ & $1000 \mathrm{sccm}$ \\
\hline Ar flow rate for thiophene monomer vapor & $100 \mathrm{sccm}$ & $100 \mathrm{sccm}$ & $\underline{100-500 \mathrm{sccm}}$ & $100 \mathrm{sccm}$ & $100 \mathrm{sccm}$ \\
\hline Polymerization process time & $10 \mathrm{~min}$ & $10 \mathrm{~min}$ & $10 \mathrm{~min}$ & $10,20,30 \mathrm{~min}$ & $10 \mathrm{~min}$ \\
\hline Results (Figures) & 3 and 4 & 5 and 6 & 8 & 9 & $10-12$ \\
\hline
\end{tabular}

\subsection{Glow Discharge Behaviors during AP Plasma Polymerization}

Three AP plasma reactors with bare-wire lengths of $2 \mathrm{~mm}, 15 \mathrm{~mm}$, and $30 \mathrm{~mm}$ were fabricated, and the discharge characteristics and polymer film uniformity were investigated according to the length of the bare electrode. In the cruciform wire electrode partially covered with the glass capillary, it was observed that the glow discharge occurred at the boundary between the bare-wire part and the capillary, based on the observation of the digital photographs and the ICCD images. When the length of the bare-wire part was very small (2 mm), AP plasma was generated only in the central bare part of the cruciform wire electrode as designed (Figure 3a). As the length of the bare-wire part increased to $15 \mathrm{~mm}$ and $30 \mathrm{~mm}$, the boundary point where discharge occurred gradually moved away from 
the center, and the discharge region was divided into fourths along the cruciform wire electrode, thereby successfully expanding the discharge volume (Figure 3b,c).

(a)
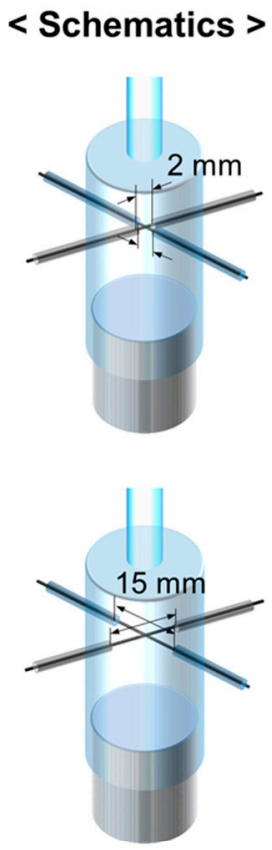

(b)

(c)

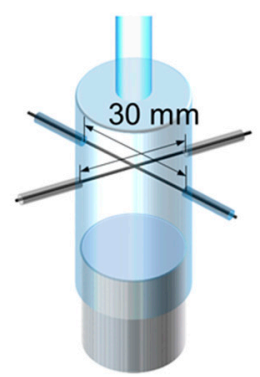

< Photographs >
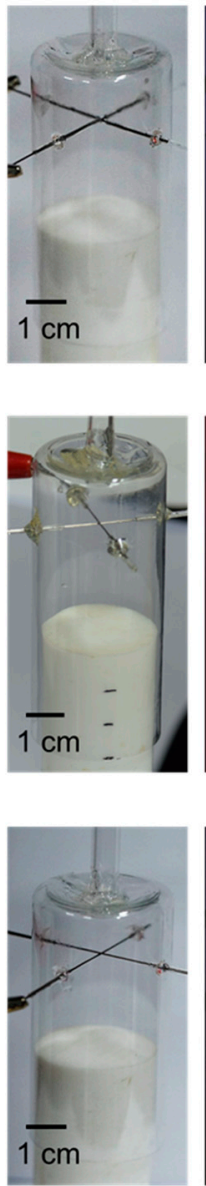

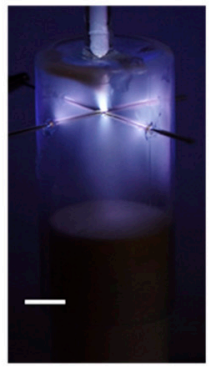

< ICCD Image >
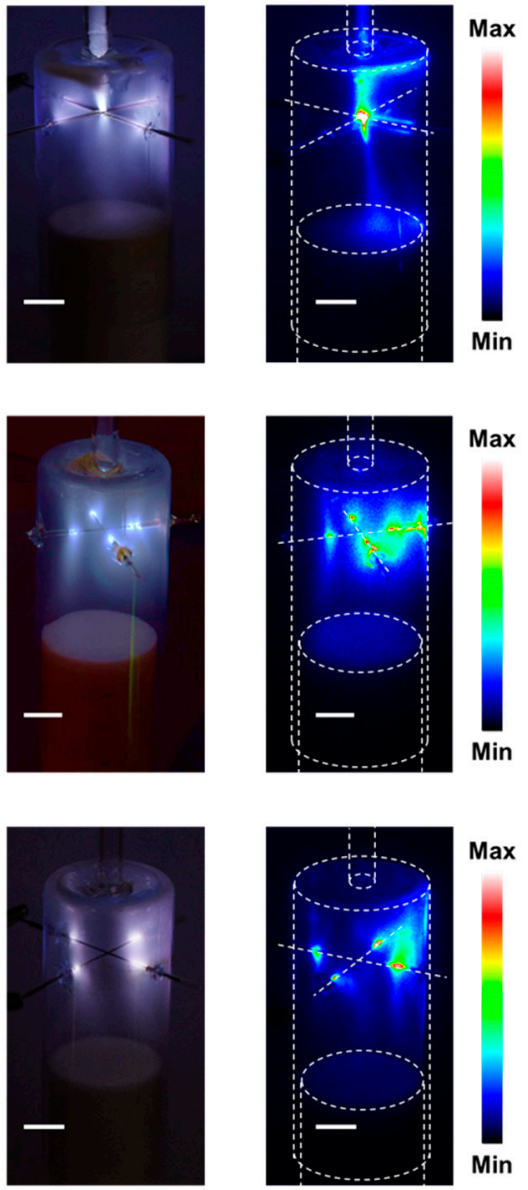

Figure 3. Optical observation of glow plasma according to length of the bare part of the cruciform wire electrode: (a) $2 \mathrm{~mm}$, (b) $15 \mathrm{~mm}$, and (c) $30 \mathrm{~mm}$.

As shown in Figure $3 a-c$, when the length of the bare-wire part was increased, the discharge initiation point shifted, but the discharge area was not widened in the AP plasma reactor. The AP plasma reactor of Figure $3 \mathrm{a}$ had an electrode structure in which the discharge was concentrated in the center, whereas in the AP plasma reactor of Figure $3 b$, four discharge-generation regions were appropriately and evenly distributed in the polymerization space. However, when the length of the bare wire was increased to $30 \mathrm{~mm}$, it was observed that the four plasma regions were very close to the sidewall of the wide tube as shown in Figure $3 c$, and the region where strong polymerization occurred also moved toward the sidewall.

\subsection{Observation of Uniformity of the PTh Film}

To investigate the uniformity of the PTh-film deposition in the entire polymerization region inside the AP plasma reactor according to the length of the bare wire, PTh films were synthesized on circular substrates with diameters of $30 \mathrm{~mm}$, which was the same size as the substrate stand, and the spatial distribution of the deposited PTh film was observed. To better observe the deposited PTh film, a transparent glass disk was used as the substrate and polymerization took place for only $5 \mathrm{~min}$ to prevent the films from being too thick in this test. As mentioned, glow plasmas successfully occurred at the four boundaries between the bare-wire parts and the covered glass capillaries in the AP plasma reactor, but 
it was difficult to make the four discharges exactly the same. Even if the experimental setup and conditions were carefully managed, the glow plasmas generated at the four boundaries could not be perfectly matched. This uneven plasma state caused the nonuniformity of the polymerized film.

As shown in Figure $4 a-c$, the uniformity of the PTh film along the length of the barewire part was investigated. In all three cases, it was observed that the resulting PTh films could not be deposited completely uniformly in the entire polymerization region. When the length of the bare-wire part was $2 \mathrm{~mm}$, the PTh film was mainly deposited near the center of the glass disk along the generated plasma shape, and thus was not uniform as a whole region (Figure 4a). When the length of the bare-wire part increased to $15 \mathrm{~mm}$, the PTh film that was deposited onto the glass disk showed an uneven pattern along the cruciform electrode due to the nonuniform plasma state (Figure $4 \mathrm{~b}$ ). In particular, when the length of the bare part was $30 \mathrm{~mm}$, the deposition regions of the PTh film did not overlap each other because the glow plasmas that were generated in the four regions were far apart from each other, resulting in a very nonuniform film (Figure 4c).
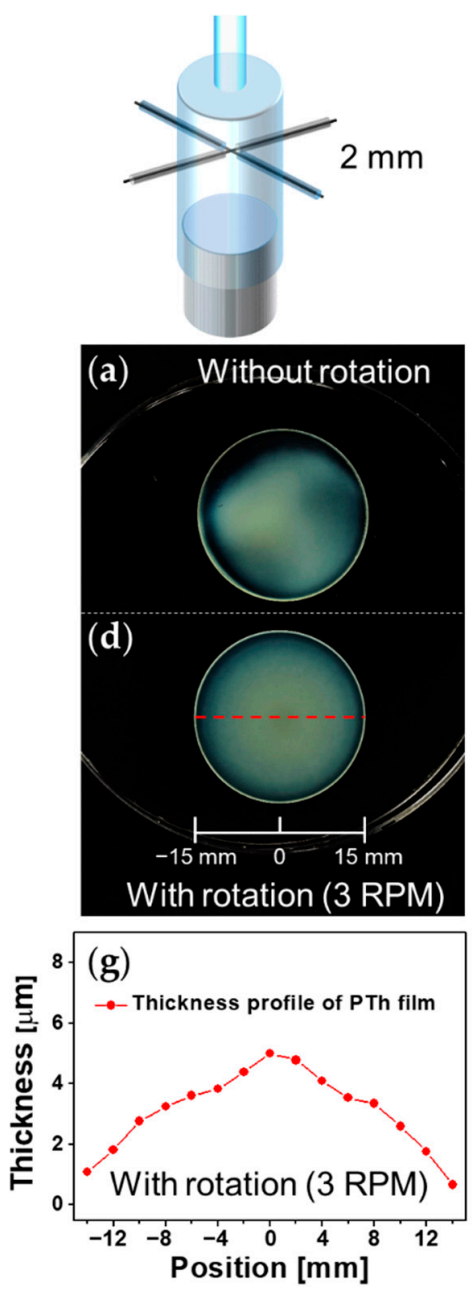
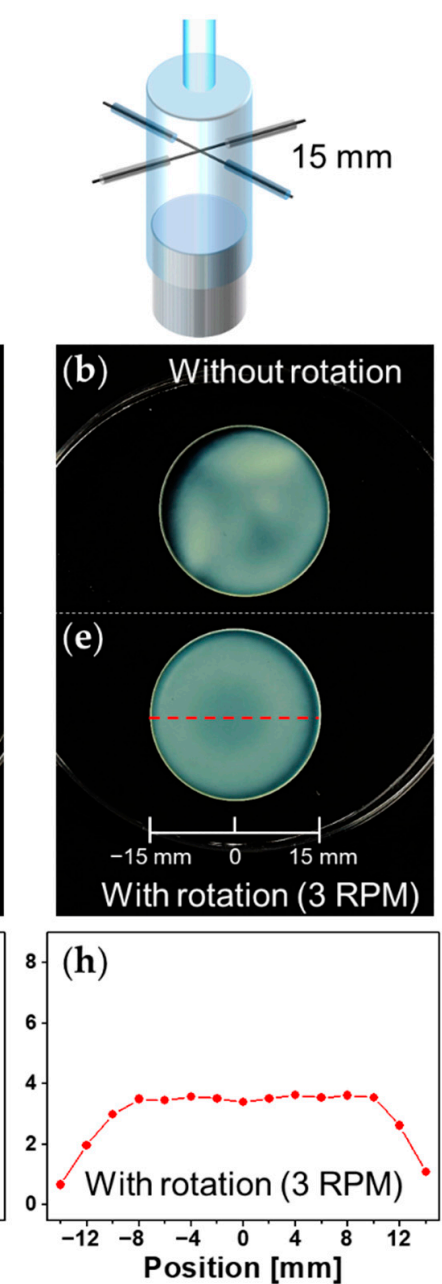
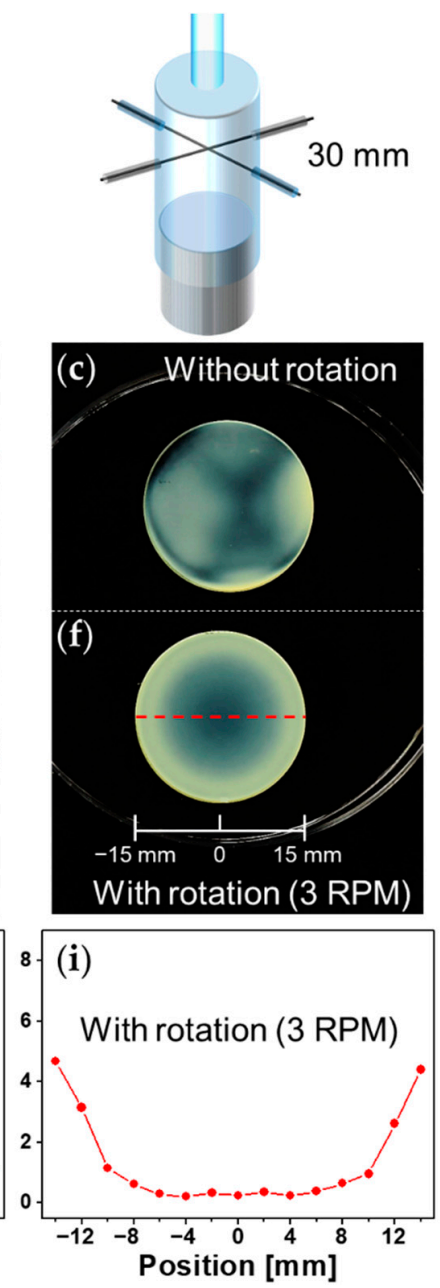

Figure 4. (a-c) Photographs of PTh films deposited onto glass disks with bare-wire lengths of (a) $2 \mathrm{~mm}$, (b) $15 \mathrm{~mm}$, and (c) $30 \mathrm{~mm}$ in an AP plasma reactor without substrate rotation; (d-f) Photographs of PTh films deposited onto glass disks with bare-wire lengths of (d) $2 \mathrm{~mm}$, (e) $15 \mathrm{~mm}$ and (f) $30 \mathrm{~mm}$ with substrate rotating at $3 \mathrm{rpm}$; $(\mathbf{g}-\mathbf{i})$ Thickness profiles of the rotated nanostructured PTh films in (d-f). 


\subsection{Substrate Rotation for Improvement of Uniformity of the PTh Film}

To resolve this issue of the nonuniformity of the PTh film, the substrate stand was rotated with a turntable during the polymerization process. The substrate stand was rotated very slowly at three revolutions per minute ( $3 \mathrm{RPM}$ ) to secure the PTh-film uniformity in the azimuthal direction and to minimize the influence that it may have on the substrate by rotation, such as centrifugal force. Therefore, the PTh films were deposited onto glass disks with the substrate rotating 15 times ( $5 \mathrm{~min} \times 3 \mathrm{RPM}$ ) as shown in Figure $4 \mathrm{~d}-\mathrm{f}$. Figure $4 \mathrm{~g}-\mathrm{i}$ show the thickness profiles of the rotated PTh films that were measured using a stylus profiler at 15 positions with an interval of $2 \mathrm{~mm}$ along the red dashed lines on the PTh films in Figure $4 \mathrm{~d}-\mathrm{f}$. As a result, the most uniform PTh film was obtained when the length of the cruciform bare wire was $15 \mathrm{~mm}$ and the substrate stand was rotated. For this reason, the morphological and chemical properties of the PTh films, which will be discussed in the next sections, were examined using this AP plasma reactor with a bare-wire length of $15 \mathrm{~mm}$ and a rotating substrate stand.

\subsection{Morphological and Chemical Properties of the Nanostructured PTh Film}

Because the cruciform powered electrode was placed parallel to the substrate in the proposed AP plasma reactor, determining the spacing between the electrode and the substrate was very critical to the growth of the nanostructured PTh film. As shown in Figure $5 \mathrm{a}-\mathrm{c}$, the FE-SEM images depict the surface morphology and film thickness of the nanostructured PTh film that was deposited onto the Si substrate according to the distance between the substrate and the electrode. When the distance between the electrode and the substrate $(D)$ was $30 \mathrm{~mm}$, a longitudinally oriented PTh nanostructure was deposited onto the Si substrate with a thickness of $7.2 \mu \mathrm{m}$, despite the short polymerization time of $10 \mathrm{~min}$ (Figure 5a,d). In general, when the polymerization reaction takes place in the discharge space and the synthesized material falls onto the substrate, it is difficult to observe vertically aligned nanostructures in the prepared polymer film. The growth of vertically aligned PTh nanostructures as shown in Figure 5 a indicated that the chemical bonding proceeded on the substrate. Therefore, the prepared nanostructured PTh film can be expected to have good adhesion to the substrate. In this regard, our research group recently examined the adhesion properties of PTh films synthesized by the AP plasma reactor [22]. As a result, the PTh films showed excellent adhesion properties on glass, Si, and PET substrates. On the other hand, as D increased, the film thickness decreased significantly (Figure 5b-d). In particular, when D was increased to $50 \mathrm{~mm}$, the surface morphology of the PTh nanostructures showed a low density and their thickness was observed to be less than $1 \mu \mathrm{m}$.

Figure 6 depicts the ATR-FTIR spectra of the PTh films, which show the characteristic peaks of PTh films as follows: C-S bending $\left(850 \mathrm{~cm}^{-1}\right), \mathrm{C}-\mathrm{H}$ in-plane deformation $\left(1039 \mathrm{~cm}^{-1}\right), \mathrm{C}-\mathrm{O}$ stretch bending $\left(1217 \mathrm{~cm}^{-1}\right), \mathrm{C}=\mathrm{C}$ asymmetric stretching vibration mode of the thiophene ring $\left(1411 \mathrm{~cm}^{-1}\right), \mathrm{C}=\mathrm{O}$ symmetric stretching vibration modes of the thiophenering $\left(1675 \mathrm{~cm}^{-1}\right.$ and $\left.1711 \mathrm{~cm}^{-1}\right)$, and $\mathrm{C}-\mathrm{H}$ stretching vibrations $\left(2931 \mathrm{~cm}^{-1}\right.$, $2977 \mathrm{~cm}^{-1}$, and $3282 \mathrm{~cm}^{-1}$ ) [29-31]. When $D$ was $30 \mathrm{~mm}$, it was observed that the intensity of most of the main peaks increased in the region of greater than $1039 \mathrm{~cm}^{-1}$ on ATR-FTIR, indicating that the contribution of the main chemical groups constituting PTh was improved. Although the PTh films were successfully deposited onto the substrate under all $D$ conditions, the quality of the PTh films changed as $D$ was varied. Consequently, we tentatively determined the optimal $D$ to be $30 \mathrm{~mm}$ based on the results shown in Figures 5 and 6. 


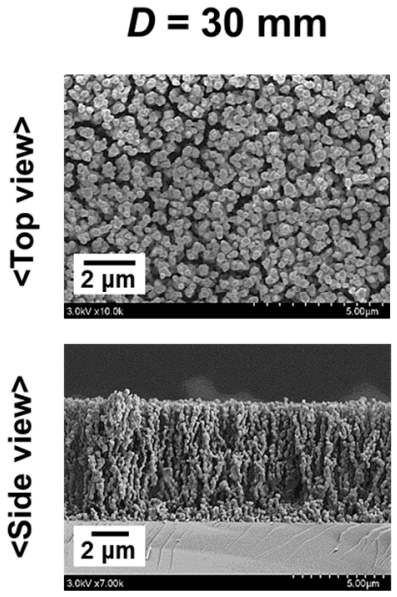

(a)
$D=\mathbf{4 0} \mathrm{mm}$
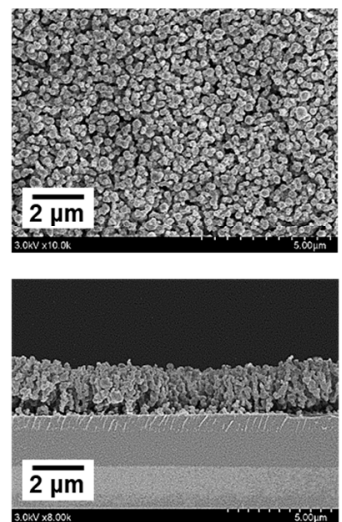

(b)
$D=50 \mathrm{~mm}$
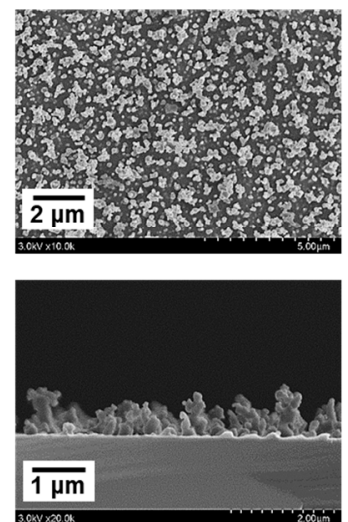

(c)

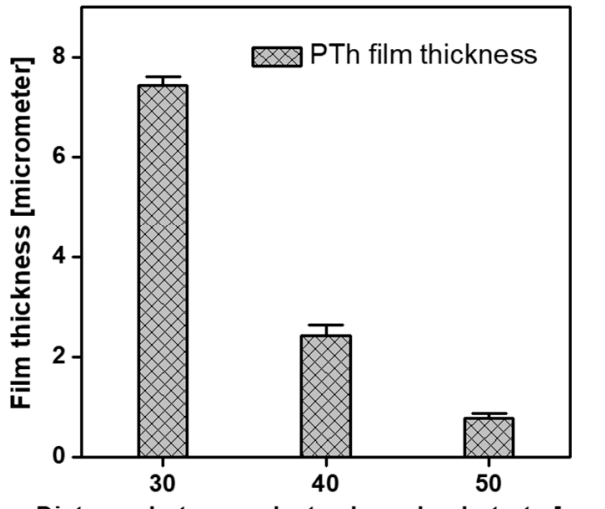

Distance between electrode and substrate [mm]

(d)

Figure 5. FE-SEM images of nanostructured PTh films deposited onto silicon substrates when the distances between the electrode and the substrate (D) were (a) $30 \mathrm{~mm}$, (b) $40 \mathrm{~mm}$, and (c) $50 \mathrm{~mm}$ and (d) comparison of thickness of PTh films.

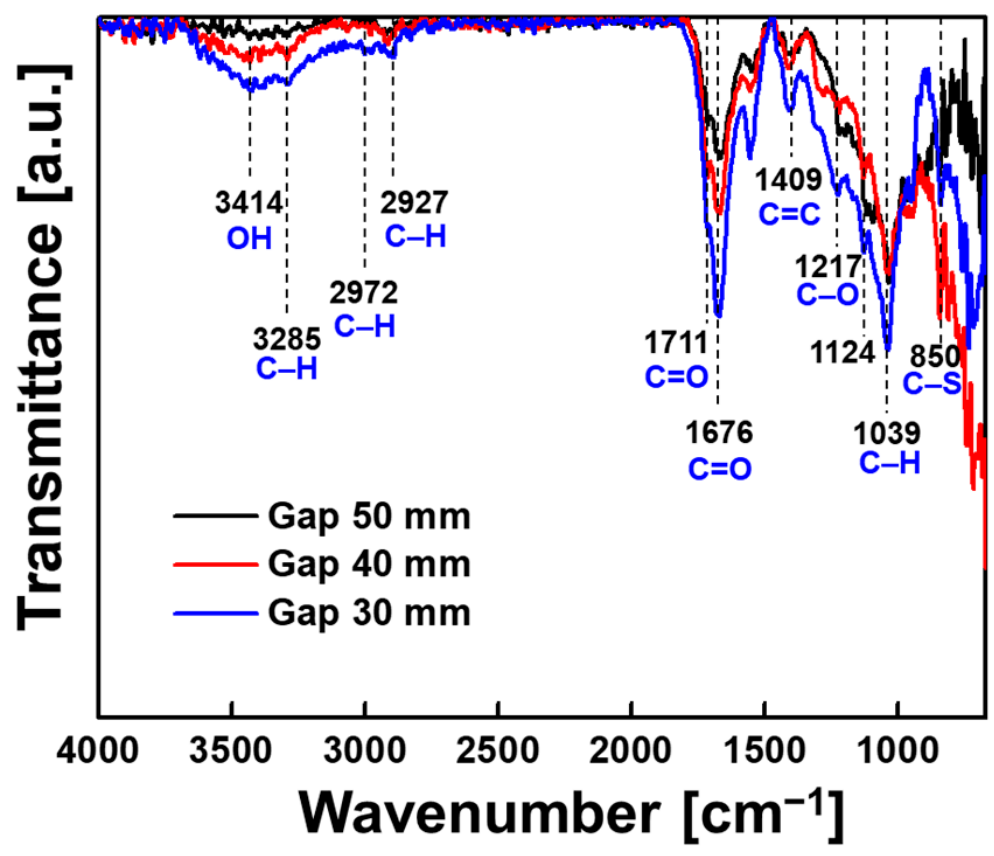

Figure 6. Comparison of ATR-FTIR spectra of deposited nanostructured PTh films when the distance between the electrode and the substrate of the plasma reactor was changed to $30 \mathrm{~mm}, 40 \mathrm{~mm}$, and $50 \mathrm{~mm}$.

Figure 7 shows the TEM images of the PTh nanoparticles generated by the proposed AP plasma reactor. The TEM sample of the PTh nanoparticle cluster was collected from the nanostructured PTh film that was deposited onto the silicon substrate when $D$ was $30 \mathrm{~mm}$ (Figure 7a). It was observed that PTh nanoparticles with diameters of approximately 20-60 nm were interconnected to form many cross-linked networks (Figure 7b), indicating that PTh nanoparticles could be effectively synthesized. 


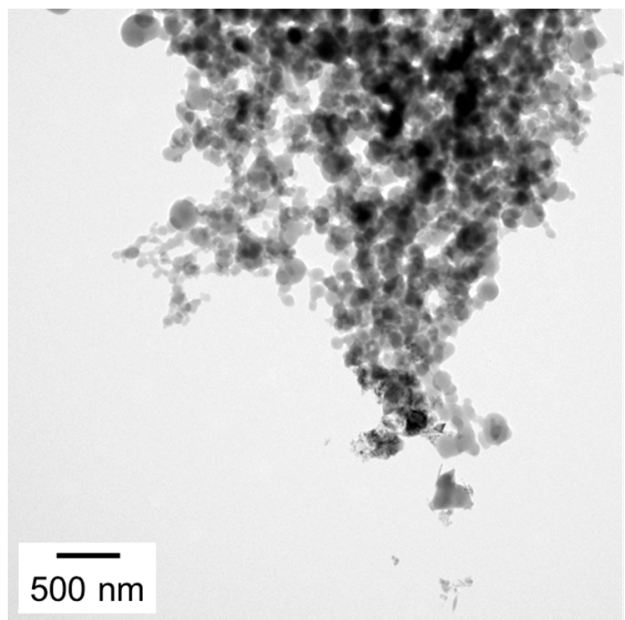

(a)

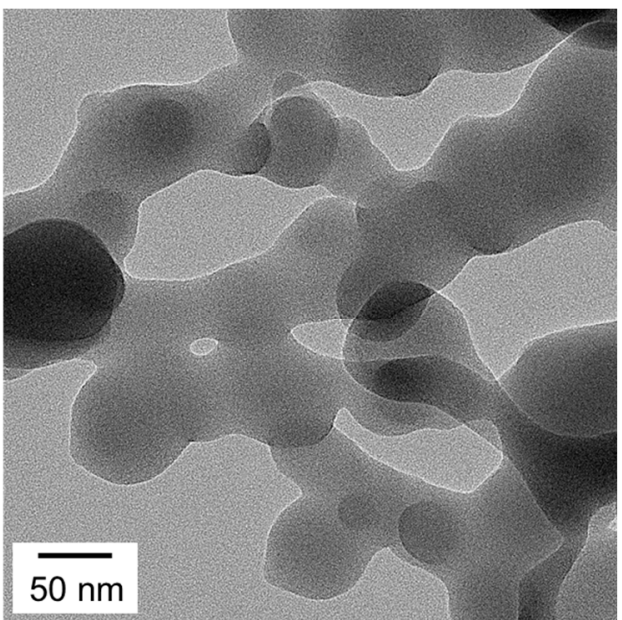

(b)

Figure 7. Transmission electron microscopy (TEM) images of PTh nanoparticles prepared via the proposed AP plasma reactor with (a) low magnification and (b) high magnification.

To determine the optimal amount of thiophene monomer under the experimental condition where $D$ is $30 \mathrm{~mm}$, the morphological properties of the deposited PTh film were investigated while changing the Ar flow applied to the bubbler containing liquid thiophene monomer. As the Ar flow for the vaporized thiophene increased from 100 to $500 \mathrm{sccm}$, the thickness of the nanostructured PTh films gradually decreased while the grain size of the PTh nanoparticles did not significantly change, as shown in Figure 8. When only the amount of vaporized thiophene molecules increased under the experimental conditions that were employed in this study, the Ar discharge became unstable, which adversely affected AP plasma polymerization and the growth rate of the PTh film. If the driving voltage of the plasma is increased, it is expected that the growth rate of the PTh film can be increased even at Ar flows for the thiophene vaporization of 200-300 sccm. However, when the driving voltage increased, the imbalance of the four discharge regions became more severe. Therefore, the Ar gas flow rate for the thiophene vapor injection was optimized to $100 \mathrm{sccm}$ under the current experimental conditions. This is because a nanostructured PTh film with a sufficient thickness of 5 to $10 \mu \mathrm{m}$ can be obtained with a short process time of 10 min while maintaining a low driving voltage.
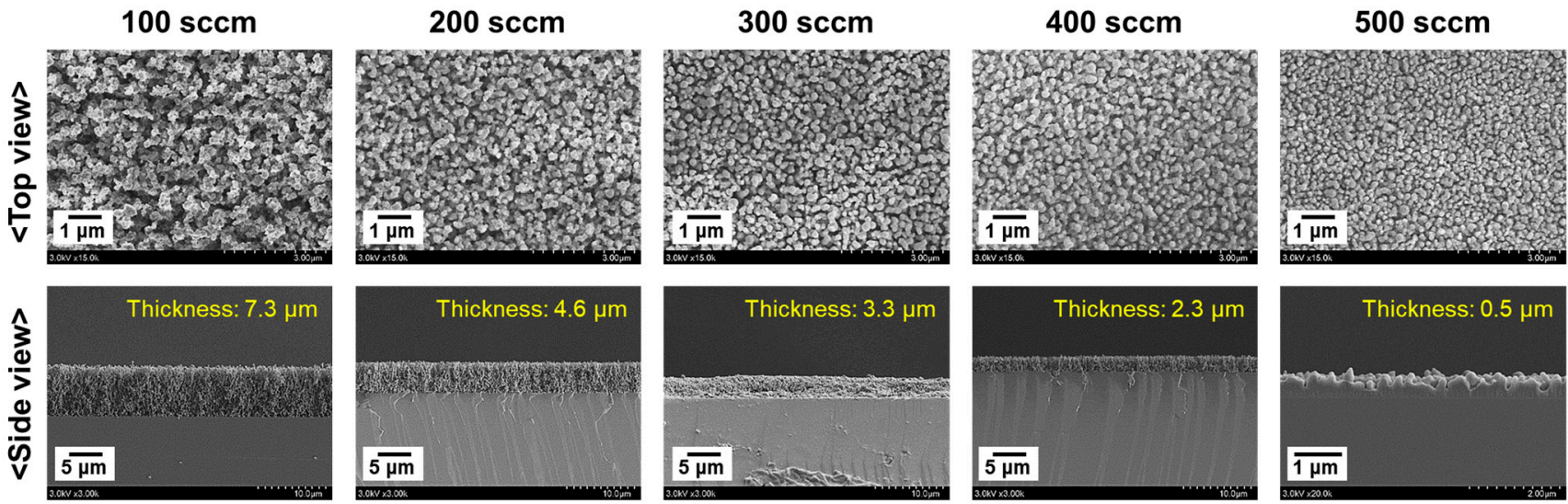

Ar flow containing thiophene vapors $(100-500$ sccm $)+$ Pure Ar flow (1000 sccm)

Figure 8. FE-SEM images of nanostructured PTh films deposited onto silicon substrates as Ar flow for vaporized thiophene increased from 100 to $500 \mathrm{sccm}$. 
At the optimal gas conditions that combined a pure Ar flow of $1000 \mathrm{sccm}$ and an Ar flow containing monomer vapor of $100 \mathrm{sccm}$, AP plasma polymerization was tested with increasing process times from 10 to $30 \mathrm{~min}$ in order to check the thickness of the nanostructured PTh films deposited onto the Si substrates (Figure 9). Under the same experimental conditions, except for the polymerization time, the grain sizes of the PTh nanoparticles remained almost unchanged, and the thicknesses of the nanostructured PTh films increased as a function of time. As the PTh nanoparticles accumulated in the vertical direction, the vertically aligned nanostructures were densely formed, then bundles of these nanoparticles formed a film on the Si substrate. After polymerization for $30 \mathrm{~min}$, a nanostructured PTh film of $35 \mu \mathrm{m}$ or higher can be obtained, confirming that the proposed polymerization process is a fast synthesis process at room temperature and AP conditions.

$10 \min$

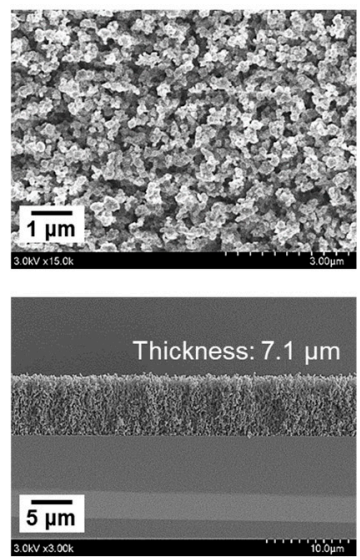

(a)
$20 \min$
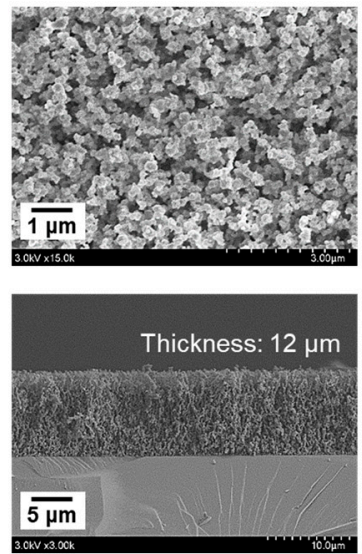

(b)
$30 \min$

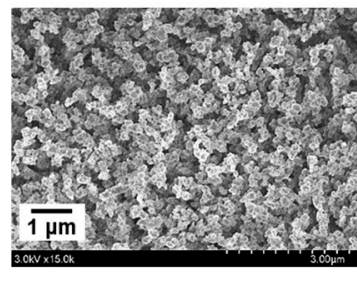

Thickness: $36 \mu \mathrm{m}$

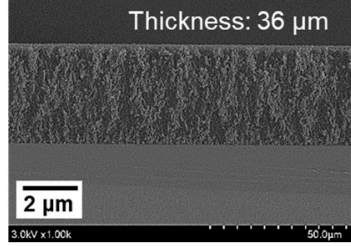

(c)

Figure 9. FE-SEM images of nanostructured PTh films as polymerization process time changed; (a) $10 \mathrm{~min}$, (b) $20 \mathrm{~min}$, and (c) $30 \mathrm{~min}$.

\subsection{Comparison of Nanostructured PTh Films Synthesized in the Plasma Remote and Coupling Modes}

As shown in Figure 5, the optimal distance between the electrode and the substrate, $D$, was tentatively determined as $30 \mathrm{~mm}$ in this study. The case where $D$ was less than $30 \mathrm{~mm}$ was examined in detail. The volume of the glow-plasma region was controlled by the volume of the plasma reactor, the presence of the counter electrode, the discharge gas (Ar) flow, the additives introduced into the plasma, and the applied voltage. By controlling the volume of the glow-plasma region and the distance between the electrode and the substrate, the generated plasma can be in contact with the substrate. In this experiment, when $D$ was $20 \mathrm{~mm}$, the glow plasma that was generated inside the guide tube may or may not have come into direct contact with the substrate due to the subtle and uncontrollable differences among the four discharges. That is probably due to the instability of the amount of vaporized thiophene monomer in this AP system. The case where the glow plasma that was generated in the discharge space contacts the substrate is called the plasma coupling mode, and the case where the plasma does not contact the substrate is called the plasma remote mode [32,33].

Because this AP plasma reactor generated glow plasmas in four places simultaneously, it could have caused slight differences among the four discharges. In addition, the proposed cruciform bare electrode potentially had an issue of discharge instability because it was directly exposed to the plasma medium. Therefore, the generated plasma volume (and length) may have varied slightly due to the relatively unstable discharge, which made it impossible to completely control the two plasma modes at a $D$ of $20 \mathrm{~mm}$. The chemical composition of the surface of PTh films that were obtained under the plasma coupling and remote conditions was analyzed using XPS results as shown in Figure 10. The XPS signals of 
the PTh films included specific signals of $\mathrm{O} 1 \mathrm{~s}(532.1 \mathrm{eV}), \mathrm{C} 1 \mathrm{~s}(285.5 \mathrm{eV})$, and S 2p (164.0 eV); these atomic concentrations are summarized in Table 3. The stoichiometric ratio of carbon to sulfur $(\mathrm{C} / \mathrm{S})$ of PTh was expected to be 4:1, because thiophene molecules have a ring structure with 4 carbons and 1 sulfur. In both the plasma remote and coupling condition, $\mathrm{C} / \mathrm{S}$ ratios of nanostructured PTh films were satisfactory at 4.1 and 4.0 , respectively.

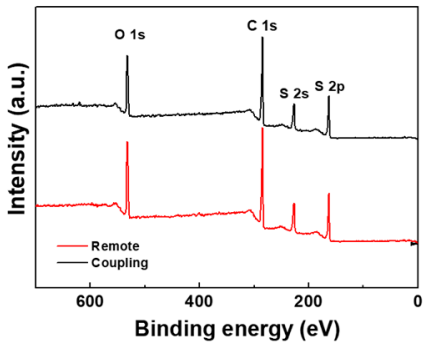

(a)
Plasma remote mode

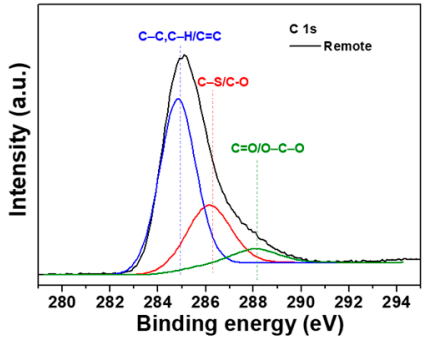

(b)

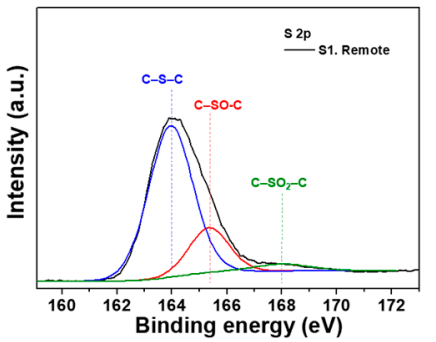

(d)
Plasma coupling mode

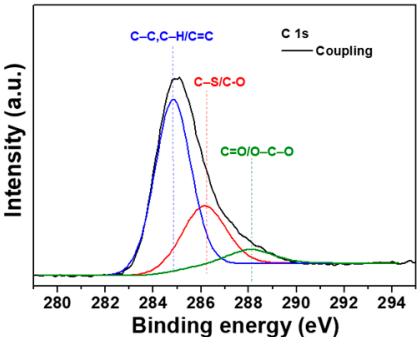

(c)

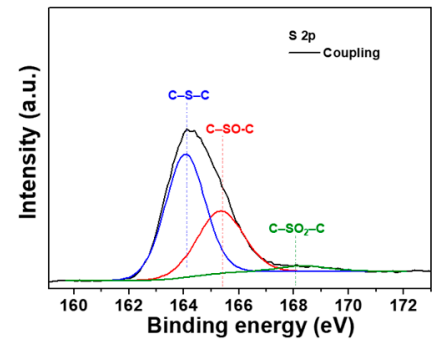

(e)

Figure 10. (a) XPS survey spectra of nanostructured PTh films synthesized under the plasma coupling and remote conditions. High-resolution XPS spectra of C 1s of PTh film prepared using the plasma (b) remote and (c) coupling modes. High-resolution XPS spectra of S 2p of PTh film prepared using the plasma (d) remote and (e) coupling modes.

Table 3. Elemental concentrations of PTh films synthesized under the plasma coupling and remote conditions.

\begin{tabular}{ccccc}
\hline Conditions & $\mathbf{C ~ ( \% )}$ & $\mathbf{O}(\mathbf{\%})$ & $\mathbf{S ~ ( \% )}$ & C/S \\
\hline Plasma remote mode & 65.4 & 18.5 & 16.1 & 4.1 \\
Plasma coupling mode & 66.6 & 17.0 & 16.5 & 4.0 \\
\hline
\end{tabular}

Figure 10b-e show the high-resolution XPS spectra of C 1s and S 2p. In Figure 10b,c, the $\mathrm{C}$ 1s peak consisted of three distinctive component peaks attributed to $\mathrm{C}-\mathrm{C}, \mathrm{C}=\mathrm{C}, \mathrm{C}-\mathrm{H}$ bonds $(284.9 \mathrm{eV}), \mathrm{C}-\mathrm{S}, \mathrm{C}-\mathrm{O}$ bonds $(286.2 \mathrm{eV})$, and $\mathrm{C}=\mathrm{O}, \mathrm{O}-\mathrm{C}-\mathrm{O}$ bonds $(288.1 \mathrm{eV})[29,34]$. The presence of $\mathrm{C}-\mathrm{O}, \mathrm{C}=\mathrm{O}$ bonds in the high-resolution $\mathrm{XPS}$ of $\mathrm{C}$ 1s were also confirmed by the FT-IR results. In Figure $10 \mathrm{~d}$,e, the $\mathrm{S} 2 \mathrm{p}$ peak could be decomposed into a $\mathrm{C}-\mathrm{S}-\mathrm{C}$ bond $(164.0 \mathrm{eV}), \mathrm{C}-\mathrm{SO}-\mathrm{C}$ bond $(165.3 \mathrm{eV})$, and $\mathrm{C}-\mathrm{SO}_{2}-\mathrm{C}$ bond $(168.2 \mathrm{eV})$, which were revealed by the aromatic ring of PTh $[34,35]$. The ratios of the deconvoluted components of $\mathrm{C}$ 1s and S 2p are summarized in Table 4. The presence of sulfur oxides in the PTh nanostructure synthesized at AP indicated that oxygen in the atmosphere was partially excited by plasma energy. Ratios of sulfur oxides ( $\mathrm{C}-\mathrm{SO}-\mathrm{C}$ and $\mathrm{C}-\mathrm{SO}_{2}-\mathrm{C}$ ) to sulfide $(\mathrm{C}-\mathrm{S}-\mathrm{C})$ were 0.33 and 0.64 for the plasma remote and coupling modes, respectively, meaning that more excited oxygen was involved in the synthesis process in the plasma coupling mode than in the plasma remote mode. Therefore, it was confirmed that more plasma energy was applied to the substrate with the PTh film in the plasma coupling mode. 
Table 4. Peak assignments and percent composition of the C 1s and S $2 p$ of PTh films prepared using the plasma remote and coupling modes.

\begin{tabular}{ccccc}
\hline \multirow{2}{*}{ Peak Assignment } & Binding & \multicolumn{2}{c}{ Composition (\%) } \\
\cline { 4 - 5 } & & Energy (eV) & Remote Mode & Coupling Mode \\
\hline \multirow{3}{*}{$\mathrm{C} 1 \mathrm{~s}$} & $\mathrm{C}-\mathrm{C}, \mathrm{C}-\mathrm{H}, \mathrm{C}=\mathrm{C}$ & 284.9 & 62.6 & 65.3 \\
& $\mathrm{C}-\mathrm{S}, \mathrm{C}-\mathrm{O}$ & 286.2 & 31.3 & 28.1 \\
& $\mathrm{C}=\mathrm{O}, \mathrm{O}-\mathrm{C}-\mathrm{O}$ & 288.1 & 6.1 & 6.6 \\
\hline \multirow{3}{*}{$\mathrm{S} 2 \mathrm{p}$} & $\mathrm{C}-\mathrm{S}-\mathrm{C}(1)$ & 164.0 & 75.2 & 60.8 \\
& $\mathrm{C}-\mathrm{SO}-\mathrm{C}(2)$ & 165.3 & 20.3 & 35.7 \\
& $\mathrm{C}-\mathrm{SO}_{2}-\mathrm{C}(3)$ & 168.2 & 4.5 & 3.5 \\
\hline
\end{tabular}

As shown in Figure 11a,b, the morphology and thickness of the nanostructured PTh film was significantly different between the plasma remote mode and plasma coupling mode. In the plasma remote mode, the PTh nanostructure grew high in the vertical direction while maintaining small grain sizes, whereas in the plasma coupling mode the PTh nanostructure had large grain sizes but did not grow high in the vertical direction. As shown in the magnified side-view FE-SEM images of Figure 11c, when the PTh nanostructure was initially grown on a Si substrate in the plasma coupling mode, it formed small nanoparticles on the substrate. When the plasma energy was transferred directly to the substrate, the grain sizes of the PTh nanostructure grew. Thus, the grain sizes gradually increased from the substrate toward the PTh-film surface. Moreover, the granularity was relatively irregular as the PTh nanoparticles grew randomly from the substrate to the film surface in the plasma coupling mode.

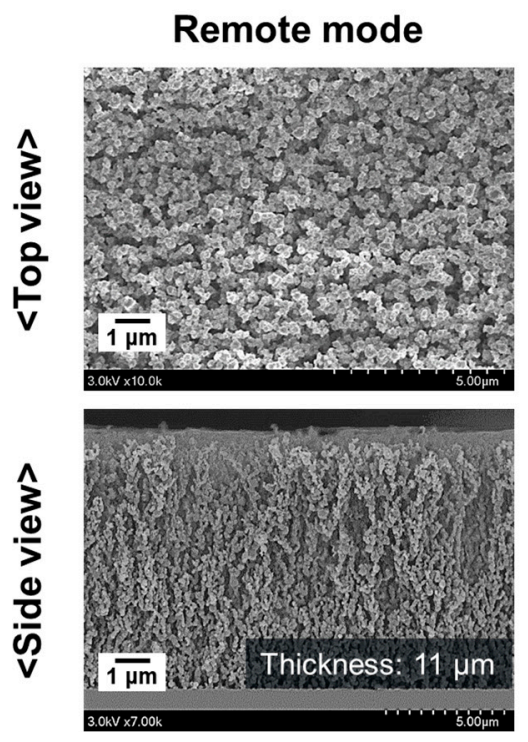

(a)

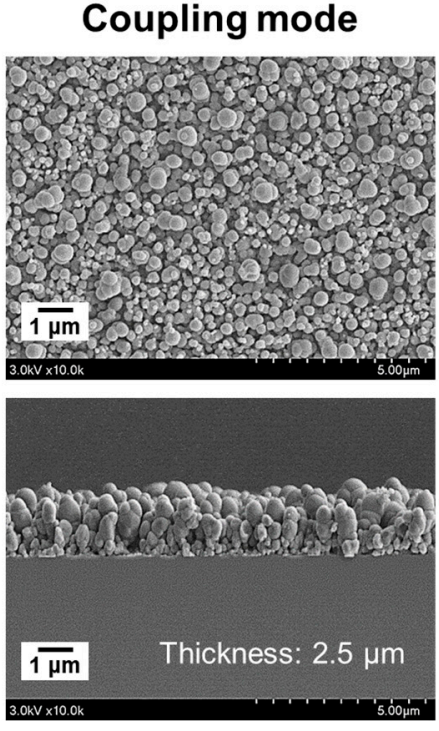

(b)
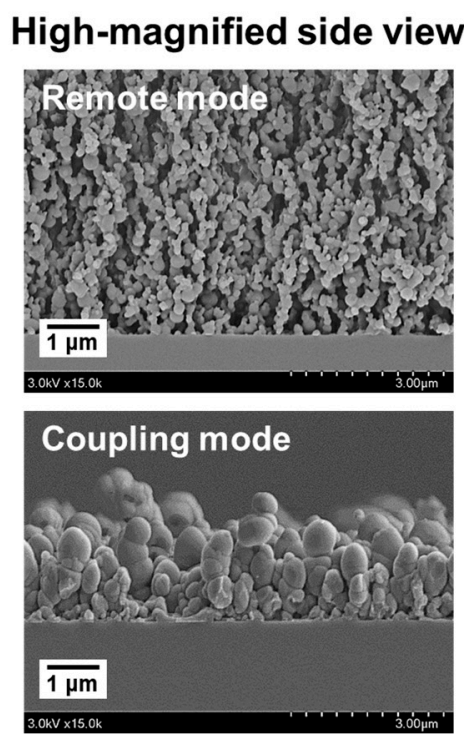

(c)

Figure 11. Comparison of FE-SEM images of plasma remote mode and coupling mode: (a) plasma remote mode, (b) plasma coupling mode, and (c) magnified side view FE-SEM images in the two different plasma modes.

In the plasma remote mode, the plasma energy was primarily used to grow the nanostructured PTh film on the substrate by bonding the PTh nanoparticles in longitudinal alignment during the polymerization process time. Conversely, in the plasma coupling mode, the plasma energy was used to increase the grain size of the PTh nanoparticles. Therefore, the thicknesses of the resulting nanostructured PTh films differed by 
more than a factor of four due to the different plasma modes occurring under the same polymerization conditions.

\subsection{Electrical Properties of Nanostructured PTh Films Synthesized in the Plasma Remote and Coupling Modes}

In order to use the fabricated nanostructured PTh film as an electrode or transducer for electronic devices, it is very important to provide electrical properties to the film. A widely used approach to making the conjugated polymers, including conductive PTh, is to dope a halogen element such as $\mathrm{HCl}$ or $\mathrm{I}_{2}$ as an electron acceptor [36-38]. An ex situ $\mathrm{I}_{2}$-doping process in which a doping procedure is performed after synthesizing a polymer film has been reported in many studies due to the advantages of the low-cost and simple process [39-41]. The $I_{2}$-doping principle and characteristics of $I_{2}$-doped conjugated polymer films have been reported in previous studies of our research group [33,42-44].

The electrical properties of conductive polymer materials are generally changed by water inside or on the surface of the nanostructure [44-46]. Thus, the resistance change of the $\mathrm{I}_{2}$-doped PTh film was measured according to the morphology of the nanostructured PTh film. Figure 12 shows the changes in the resistance of $\mathrm{I}_{2}$-doped PTh films synthesized in plasma coupling and remote modes with exposure time in ambient air. The measurement limit of the resistance of the $\mathrm{I}_{2}$-doped PTh film was $50 \mathrm{M} \Omega$, and if it exceeded this limit then it was considered to have infinite resistance. In the plasma remote mode, the initial resistance of the $I_{2}$-doped PTh film was measured to be $65 \mathrm{k} \Omega$, and the resistance gradually increased in the atmosphere and reached the measurement limit, $50 \mathrm{M} \Omega$, after $60 \mathrm{~min}$. However, in the plasma coupling mode, the initial resistance was measured to be $150 \mathrm{k} \Omega$ and the resistance rapidly increased and reached the measurement limit in only $20 \mathrm{~min}$. The dense and thick $\mathrm{I}_{2}$-doped PTh film that was obtained in the plasma remote mode was less affected by hydration under ambient air and had better stability to resistance changes. It was confirmed that the characteristics of the resistance change according to exposure time were in good agreement with the previously reported results [33]. The $\mathrm{I}_{2}$-doped PTh film with time-varying resistance properties can be expected to have a constant electrical conductivity when encapsulated with a sealing material to prevent the access of moisture and oxygen [42].

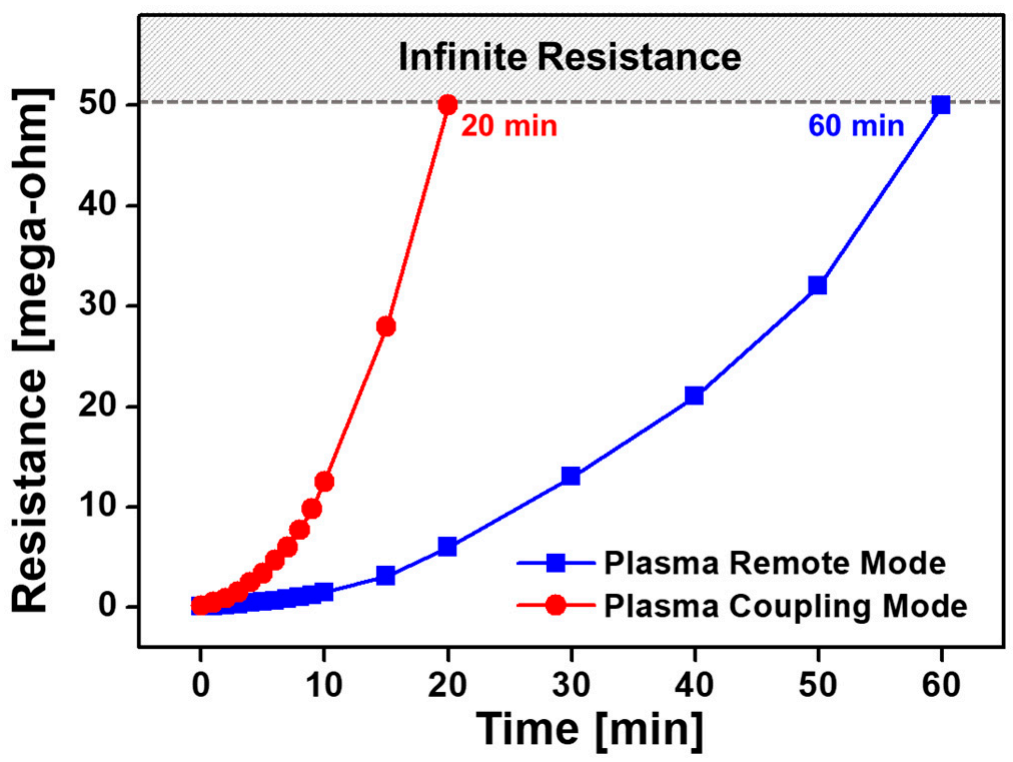

Figure 12. Changes in electrical resistance of iodine-doped PTh films on IDE substrates synthesized in plasma coupling and remote modes. 


\section{Conclusions}

In this study, we proposed an AP plasma reactor with a cruciform electrode structure that had the advantage of an easy spatial expansion of the plasma medium, and investigated the morphological and chemical properties of the deposited PTh film using this plasm reactor. In the cruciform wire electrode partially covered with glass capillary tubes, the discharge occurred at the boundary where the covered capillary tubes ended. Thus, as the bare electrode part became longer, the boundary points where the discharge occurred gradually moved away from the center and the discharge region was divided into fourths in one AP plasma reactor. As a result, the glow-plasma medium expanded spatially. Even with careful management of the experimental setup and conditions, the plasma properties of the four boundaries cannot be completely matched, leading to the non-uniform deposition of the nanostructured PTh film. However, the uniformity of the nanostructured PTh film could be considerably improved by rotating the substrate stand using a turntable. The study of discharge characteristics and PTh-film properties according to the length of the bare electrode and the substrate rotation will be important experimental data for the development of advanced AP-plasma-polymerization methods that are capable of depositing a large-area polymer film using multiple electrodes. In addition, further studies on the characterization of AP glow plasmas that are generated from diverse cruciform or reticulated electrode structures will provide important clues to the infinite spatial scalability of polymer deposition under AP conditions.

Supplementary Materials: The following supporting information can be downloaded at: https: / / www.mdpi.com/article/10.3390/nano12010032/s1, Figure S1: Applied waveforms of (a) voltage, (b) total current, and (c) plasma discharge current under the conditions of Table 1.

Author Contributions: Conceptualization, J.Y.K. and H.-S.T.; methodology, J.Y.K., H.J.J. and E.Y.J.; software, E.Y.J.; validation, G.T.B., E.Y.J. and C.-S.P.; formal analysis, J.Y.K., H.J.J., C.-S.P. and E.Y.J.; investigation, J.Y.K., H.J.J., E.Y.J. and H.-S.T.; resources, J.Y.K. and H.-S.T.; data curation, J.Y.K., H.J.J., G.T.B. and E.Y.J.; writing—original draft preparation, J.Y.K., H.J.J., C.-S.P., E.Y.J. and H.-S.T.; writingreview and editing, J.Y.K., H.J.J., E.Y.J. and H.-S.T.; visualization, J.Y.K. and E.Y.J.; supervision, E.Y.J. and H.-S.T.; project administration, J.Y.K. and H.-S.T.; funding acquisition, J.Y.K. and H.-S.T. All authors have read and agreed to the published version of the manuscript.

Funding: This research was supported by two National Research Foundation of Korea (NRF) grants funded by the Korean government (Ministry of Education; MOE) (No. 2020R1I1A3071693 and No. 2021R1I1A3049028) and by funding from the BK21 FOUR project funded by the Ministry of Education, Korea (4199990113966).

Institutional Review Board Statement: Not applicable.

Informed Consent Statement: Not applicable.

Data Availability Statement: Not applicable.

Acknowledgments: The authors would like to thank Sang-Geul Lee and Weon-Sik Chae at the Korea Basic Science Institute (Daegu) for their useful discussions and for providing ATR-FTIR data.

Conflicts of Interest: The authors declare no conflict of interest.

\section{References}

1. Friedrich, J. Mechanisms of plasma polymerization-Reviewed from a chemical point of view. Plasma Process. Polym. 2011, 8, 783-802. [CrossRef]

2. Bhatt, S.; Pulpytel, J.; Aref-Khonsari, F. Low and atmospheric plasma polymerisation of nanocoatings for bio-applications. Surf. Innov. 2015, 3, 63-83. [CrossRef]

3. Michel, M.; Bour, J.; Petersen, J.; Arnoult, C.; Ettingshausen, F.; Roth, C.; Ruch, D. Atmospheric plasma deposition: A new pathway in the design of conducting polymer-based anodes for hydrogen. Fuel Cells 2010, 10, 932-937. [CrossRef]

4. Uygun, A.; Oksuz, L.; Yavuz, A.G.; Guleç, A.; Sen, S. Characteristics of nanocomposite films deposited by atmospheric pressure uniform RF glow plasma. Curr. Appl. Phys. 2011, 11, 250-254. [CrossRef]

5. Chen, K.S.; Liao, S.C.; Lin, S.W.; Tsao, S.H.; Ting, T.H.; Inagaki, N.; Wu, H.M.; Chen, W.Y. The film deposition via atmospheric pressure plasma from ethanol and He mixing gases. Surf. Coat. Technol. 2013, 231, 408-411. [CrossRef] 
6. Phillips, J.; Luhrs, C.C.; Richard, M. Review: Engineering particles using the aerosol-through-plasma method. IEEE Trans. Plasma Sci. 2009, 37, 726-739. [CrossRef]

7. Penkov, O.V.; Khadem, M.; Lim, W.-S.; Kim, D.-E. A review of recent applications of atmospheric pressure plasma jets for materials processing. J. Coat. Technol. Res. 2015, 12, 225-235. [CrossRef]

8. Becker, K.H.; Schoenbach, K.H.; Eden, J.G. Microplasmas and applications. J. Phys. D Appl. Phys. 2006, 39, R55-R70. [CrossRef]

9. Khelifa, F.; Ershov, S.; Habibi, Y.; Snyders, R.; Dubois, P. Free-radical-induced grafting from plasma polymer surfaces. Chem. Rev. 2016, 116, 3975-4005. [CrossRef] [PubMed]

10. Akishev, Y.; Grushin, M.; Karalnik, V.; Kochetov, I.; Napartovich, A.; Trushkin, N. Generation of atmospheric pressure nonthermal plasma by diffusive and constricted discharges in air and nitrogen at the rest and flow. J. Phys. Conf. Ser. 2010, 257, 012014. [CrossRef]

11. Šimončicová, J.; Kryštofová, S.; Medvecká, V.; Ďurišová, K.; Kaliňáková, B. Technical applications of plasma treatments: Current state and perspectives. Appl. Microbiol. Biotechnol. 2019, 103, 5117-5129. [CrossRef] [PubMed]

12. Haertel, B.; von Woedtke, T.; Weltmann, K.-D.; Lindequist, U. Non-thermal atmospheric-pressure plasma possible application in wound healing. Biomol. Ther. 2014, 22, 477-490. [CrossRef]

13. Graves, D.B. The emerging role of reactive oxygen and nitrogen species in redox biology and some implications for plasma applications to medicine and biology. J. Phys. D Appl. Phys. 2012, 45, 263001. [CrossRef]

14. Tendero, C.; Tixier, C.; Tristant, P.; Desmaison, J.; Leprince, P. Atmospheric pressure plasmas: A review. Spectrochim. Acta B 2006, 61, 2-30. [CrossRef]

15. Jang, H.J.; Jung, E.Y.; Parsons, T.; Tae, H.-S.; Park, C.-S. A review of plasma synthesis methods for polymer films and nanoparticles under atmospheric pressure conditions. Polymers 2021, 13, 2267. [CrossRef]

16. Jalaber, V.; Del Frari, D.; De Winter, J.; Mehennaoui, K.; Planchon, S.; Choquet, P.; Detrembleur, C.; Moreno-Couranjou, M. Atmospheric aerosol assisted pulsed plasma polymerization: An environmentally friendly technique for tunable catechol-bearing thin films. Front. Chem. 2019, 7, 183. [CrossRef]

17. Petersen, J.; Becker, C.; Fouquet, T.; Addiego, F.; Toniazzo, V.; Dinia, A.; Ruch, D. Nano-ordered thin films achieved by soft atmospheric plasma polymerization. RSC Adv. 2013, 3, 4416-4424. [CrossRef]

18. Sajeeb, U.S.; Mathai, C.J.; Sarvanan, S.; Ashokan, R.R.; Venkatachalam, S.; Anantharaman, M.R. On the optical and electrical properties of $\mathrm{rf}$ and a.c. plasma polymerized aniline thin films. Bull. Mater. Sci. 2006, 29, 159-163.

19. Park, C.-S.; Kim, D.H.; Shin, B.J.; Tae, H.-S. Synthesis and characterization of nanofibrous polyaniline thin film prepared by novel atmospheric pressure plasma polymerization technique. Materials 2016, 9, 39. [CrossRef] [PubMed]

20. Park, C.-S.; Kim, D.H.; Shin, B.J.; Kim, D.; Lee, H.-K.; Tae, H.-S. Conductive polymer synthesis with single-crystallinity via a novel plasma polymerization technique for gas sensor applications. Materials 2016, 9, 812. [CrossRef] [PubMed]

21. Kim, D.H.; Park, C.-S.; Kim, W.H.; Shin, B.J.; Hong, J.G.; Park, T.S.; Seo, J.H.; Tae, H.-S. Influences of guide-tube and bluff-body on advanced atmospheric pressure plasma source for single-crystalline polymer nanoparticle synthesis at low temperature. Phys. Plasmas 2017, 24, 23506. [CrossRef]

22. Jang, H.J.; Park, C.-S.; Jung, E.Y.; Bae, G.T.; Shin, B.J.; Tae, H.-S. Synthesis and properties of thiophene and aniline copolymer using atmospheric pressure plasma jets copolymerization technique. Polymers 2020, 12, 2225. [CrossRef] [PubMed]

23. Kim, J.Y.; Iqbal, S.; Jang, H.J.; Jung, E.Y.; Bae, G.T.; Park, C.-S.; Shin, B.J.; Tae, H.-S. Transparent polyaniline thin film synthesized using a low-voltage-driven atmospheric pressure plasma reactor. Materials 2021, 14, 1278. [CrossRef]

24. Kim, J.Y.; Iqbal, S.; Jang, H.J.; Jung, E.Y.; Bae, G.T.; Park, C.-S.; Tae, H.-S. In-situ iodine doping characteristics of conductive polyaniline film polymerized by low-voltage-driven atmospheric pressure plasma. Polymers 2021, 13, 418. [CrossRef]

25. Van Vrekhem, S.; Morent, R.; Geyter, N.D. Deposition of a PMMA coating with an atmospheric pressure plasma jet. J. Coat. Technol. Res. 2018, 15, 679-690. [CrossRef]

26. Srisonphan, S.; Ruangwong, K.; Thammaniphit, C. Localized electric field enhanced streamer cold plasma interaction on biological curved surfaces and its shadow effect. Plasma Chem. Plasma Process. 2020, 40, 1253-1265. [CrossRef]

27. Li, J.; Lei, B.; Wang, J.; Xu, B.; Ran, S.; Wang, Y.; Zhang, T.; Tang, J.; Zhao, W.; Duan, Y. Atmospheric diffuse plasma jet formation from positive-pseudo-streamer and negative pulseless glow discharges. Commun. Phys. 2021, 4, 64. [CrossRef]

28. Vanraes, P.; Nikiforov, A.; Bogaerts, A.; Leys, C. Study of an AC dielectric barrier single micro-discharge filament over a water film. Sci. Rep. 2018, 8, 10919. [CrossRef]

29. Teslaru, T.; Topala, I.; Dobromir, M.; Pohoata, V.; Curecheriu, L.; Dumitrascu, N. Polythiophene films obtained by polymerization under atmospheric pressure plasma conditions. Mater. Chem. Phys. 2016, 169, 120-127. [CrossRef]

30. Zhang, H.; Hu, L.; Tu, J.; Jiao, S. Electrochemically assembling of polythiophene film in ionic liquids(ILs) microemulsions and its application in an electrochemical capacitor. Electrochim. Acta 2014, 120, 122-127. [CrossRef]

31. Navale, S.T.; Mane, A.T.; Khuspe, G.D.; Chougule, M.A.; Patil, V.B. Room temperature $\mathrm{NO}_{2}$ sensing properties of polythiophene films. Synth. Met. 2014, 195, 228-233. [CrossRef]

32. Kim, D.H.; Park, C.-S.; Jung, E.Y.; Kum, D.S.; Kim, J.Y.; Kim, D.; Bae, G.T.; Cho, B.-G.; Shin, B.J.; Lee, D.H.; et al. Experimental study on atmospheric pressure plasma polymerized conducting polymer under coupling and remote conditions. Mol. Cryst. Liq. Cryst. 2018, 663, 108-114. [CrossRef] 
33. Kim, D.H.; Park, C.-S.; Jung, E.Y.; Shin, B.J.; Kim, J.Y.; Bae, G.T.; Jang, H.J.; Cho, B.-G.; Tae, H.-S. Effects of iodine dopant on atmospheric pressure plasma polymerized pyrrole in remote and coupling methods. Mol. Cryst. Liq. Cryst. 2018, 677, 135-142. [CrossRef]

34. Ji, H.; Wang, T.; Liu, Y.; Lu, C.; Yang, G.; Ding, W.; Hou, W. A novel approach for sulfur-doped hierarchically porous carbon with excellent capacitance for electrochemical energy storage. Chem. Commun. 2016, 52, 12725-12728. [CrossRef] [PubMed]

35. Zhu, M.; Zhang, W.; Li, Y.; Gai, L.; Zhou, J.; Ma, W. Multishell structured magnetic nanocomposites carrying a copolymer of pyrrole-thiophene for highly selective Au(III) recovery. J. Mater. Chem. A 2016, 4, 19060-19069. [CrossRef]

36. Elmas, S.; Beelders, W.; Nash, J.; Macdonald, T.J.; Jasieniak, M.; Griesser, H.J.; Nann, T. Photo-doping of plasma-deposited polyaniline (PAni). RSC Adv. 2016, 6, 70691-70699. [CrossRef]

37. Fan, L.; Xu, X. A simple strategy to enhance electrical conductivity of nanotube-conjugate polymer composites via io-dine-doping. RSC Adv. 2015, 5, 78104-78108. [CrossRef]

38. Pron, A.; Rannou, P. Processible conjugated polymers: From organic semiconductors to organic metals and superconductors. Prog. Polym. Sci. 2002, 27, 135-190. [CrossRef]

39. Mathai, C.J.; Saravanan, S.; Anatharaman, M.R.; Venkitachalam, S.; Jayalekshmi, S. Effect of iodine doping on the bandgap of plasma polymerized aniline thin films. J. Phys. D Appl. Phys. 2002, 35, 2206-2210. [CrossRef]

40. Wang, J.; Neoh, K.G.; Kang, E.T. Comparative study of chemically synthesized and plasma polymerized pyrrole and thio-phene thin films. Thin Solid Films 2004, 446, 205-217. [CrossRef]

41. Silverstein, M.S.; Visoly-Fisher, I. Plasma polymerized thiophene: Molecular structure and electrical properties. Polymer 2002, 43, 11-20. [CrossRef]

42. Kim, J.-Y.; Jang, H.-J.; Jung, E.; Bae, G.; Lee, S.; Park, C.-S.; Shin, B.; Tae, H.-S. Improvement of the uniformity and electrical properties of polyaniline nanocomposite film by addition of auxiliary gases during atmospheric pressure plasma polymerization Nanomaterials 2021, 11, 2315. [CrossRef]

43. Park, C.-S.; Jung, E.Y.; Kim, D.H.; Kim, D.Y.; Lee, H.-K.; Shin, B.J.; Lee, D.H.; Tae, H.-S. Atmospheric pressure plasma polymerization synthesis and characterization of polyaniline films doped with and without iodine. Materials 2017, 10, 1272. [CrossRef] [PubMed]

44. Park, C.-S.; Kim, D.Y.; Kim, D.H.; Lee, H.-K.; Shin, B.J.; Tae, H.-S. Humidity-independent conducting polyaniline films synthesized using advanced atmospheric pressure plasma polymerization with in-situ iodine doping. Appl. Phys. Lett. 2017, 110, 33502. [CrossRef]

45. Ameen, S.; Song, M.; Kim, D.-G.; Im, Y.-B.; Seo, H.-K.; Kim, Y.S.; Shin, H.-S. Iodine doped polyaniline thin film for hetero-structure devices via PECVD technique: Morphological, structural, and electrical properties. Macromol. Res. 2012, 20, 30-36. [CrossRef]

46. Mohammed, M.K.A.; Al-Mousoi, A.K.; Khalaf, H.A. Deposition of multi-layer graphene (MLG) film on glass slide by flame synthesis technique. Optik 2016, 127, 9848-9852. [CrossRef] 